\title{
Wavelet-Galerkin Method and Some Numerical Method for Lane-Emden Type Differential Equation
}

\author{
Jafar Biazar*, Fereshteh Goldoust \\ Department of Applied Mathematics, Faculty of Mathematical Sciences, University of Guilan, Guilan, Rasht, Iran \\ *Corresponding author: biazar@guilan.ac.ir
}

Received August 09, 2013; Revised September 21, 2013; Accepted October 08, 2013

\begin{abstract}
In this paper, we will compare the performance of Adomian decomposition method and the waveletGalerkin method applied to the Lane-Emden type differential equation. The Galerkin Wavelet method (GWM), which is known as a numerical approach is used for the Lane- Emden equation, as an initial value problem. This approach consists of using integral operator, to convert the Lane- Emden equation in to an integral equation, then applying Galerkin Wavelet method to solve the resulted integral equation. The properties of Galerkin Wavelet method (GWM) and the Adomian Decomposition Method are also addressed. Although the Adomian decomposition solution required slightly more computational effort than the wavelet-Galerkin solution, it resulted in more accurate results than the wavelet-Galerkin method. To illustrate the methods two examples are provided and the results are in good agreement with exact solution.
\end{abstract}

Keywords: Galerkin Wavelet, Adomian Decomposition method, Lane-Emden equation, integral equations

Cite This Article: Jafar Biazar, and Fereshteh Goldoust, "Wavelet-Galerkin Method and Some Numerical Method for Lane-Emden Type Differential Equation.” American Journal of Applied Mathematics and Statistics 1, no. 5 (2013): 83-86. doi: 10.12691/ajams-1-5-1.

\section{Introduction}

In recent years, the studies of initial value problems, presented as second order ordinary differential equations (ODEs) have attached the attention of many mathematicians and physicists. One of the known equations of this type is the Lane- Emden equation, formulated as the fallowing

$$
u^{\prime \prime}(x)+\frac{\alpha}{x} u^{\prime}(x)+f(x, u)=g(x), 0<x \leq 1, \alpha \geq 0
$$

With initial conditions

$$
u(0)=A, u^{\prime}(0)=B,
$$

Where $A, B$ are constant, $f(x, u)$ is a continuous real valued function, and $g(x) \in C[0,1]$. Eq. (1) has been used to model several phenomena in mathematical physics and astrophysics such as the theory of staller structure, the thermal behavior of a spherical cloud of gas [1,2,3].

Several methods, for solving the Lane-Emden equation are known. A discussion of formulation of some of these models and the physical structure of the solutions can be found in [4,5,6]. Most algorithms currently in use for handling the Lane-Emden type problems are based on series solutions. Wavelet theory is a relatively new and an emerging area in mathematical research. It has been applied in a wide range of engineering disciplines; particularly, wavelets are successfully used in signal analysis for wave form representation and segmentations, time-frequency analysis and fast algorithms for easy implementation [7].

\section{Wavelets and Galerkin Wavelets}

Wavelets constitute a family of functions constructed from dilation and translation of a single function called the mother wavelet. When the dilation parameter $a$ and the translation parameter $b$, vary continuously we have the following family of continuous wavelets [8]:

$$
\phi_{a, b}(t)=|a|^{-\frac{1}{2}} \phi\left(\frac{t-b}{a}\right), \quad a, b \in R, a \neq 0 .
$$

If we restrict the parameters $a$ and $b$ to discrete values as $a=a_{0}^{-k}, b=n b_{0} a_{0}^{-k}, a_{0}>1, b_{0}>0$ where $n, k$ are positive integers, we have the following family of discrete wavelets.

$$
\phi_{k, n}(t)=\left|a_{0}\right|^{\frac{k}{2}} \phi\left(a_{0}^{k} t-n b_{0}\right)
$$

Where $\phi_{k, n}(t)$, a wavelet basis in $L^{2}(R)$. In particular, if $a_{0}=2, b_{0}=1$, then $\phi_{k, n}(t)$ forms an orthonormal basis, [8].

A Multi-resolution analysis of $L^{2}(R)$ is defined as a sequence of closed subspaces $v_{j}$ with the following properties, [4]

1) $v_{j} \subset v_{j+1} \quad \forall j \in Z$, 
2) $f(x) \in v_{j} \Leftrightarrow f(2 x) \in v_{j+1}$,

3) $f(x) \in v_{0} \Leftrightarrow f(x+1) \in v_{0}$,

4) $\bigcup_{j \in Z} v_{j}$ Are dens in $L^{2}(R), \bigcap_{j \in Z} v_{j}=0$.

The existence of a scaling function $\varphi(x)$ is required for which the translate generate a basis in each $v_{j}$ i.e.

With

$$
v_{j}=\overline{\operatorname{span}}\left\{\varphi_{j i}\right\}_{i \in Z}
$$

$$
\varphi_{j i}=2^{\frac{j}{2}} \varphi\left(2^{j} x-i\right) i, j \in Z .
$$

In the classical case this basis is orthonormal, so that

$$
\left\langle\varphi_{j i}, \varphi_{j k}\right\rangle_{R}=\delta_{i k}
$$

Where $\langle f, g\rangle=\int_{-\infty}^{\infty} f(x) \overline{g(x)} d x$, is the usual inner product.

Let $w_{j}$ denote a subspace complementing the subspace $v_{j}$ in $v_{j+1}$ i.e. $v_{j+1}=v_{j} \oplus w_{j}$.

Each element of $v_{j+1}$ can be uniquely written as the sum of two elements, one in $v_{j}$ and the other in $w_{j}$, which contains the details needed to pass from an approximation at the level $j$ to an approximation at the level $j+1$.

Based on the function $\varphi(x)$. One can find $\phi(x)$, the socalled mother wavelet, of which the translates and dilates constitute orthonormal bases of the spaces $w_{j}, w_{j}=\overline{\operatorname{span}}\left\{\varphi_{j i}\right\}_{i \in Z}$, generated by the following wavelets

$$
\varphi_{j i}=2^{\frac{j}{2}} \phi\left(2^{j} x-i\right) i, j \in Z
$$

Each function $f \in L^{2}(R)$, can now be expressed as

$$
f(x)=\sum_{i \in Z} c_{j 0 i} \varphi_{j 0 i}+\sum_{j=j_{0}}^{\infty} \sum_{i \in Z} d_{j i} \phi_{j i}(x),
$$

Where

$$
c_{j i}=\left\langle f, \varphi_{j i}\right\rangle_{R}, d_{j i}=\left\langle f, \phi_{j i}\right\rangle_{R}
$$

Of course, in numerical application the summation (4) are truncated, which corresponds to the projection of $f$ into a subspace $v_{j} \subset L^{2}(R)$. as otherwise by construction,

$$
\begin{aligned}
& \left\langle\phi_{j i}, \phi_{l k}\right\rangle=\delta_{j l} \delta_{i k}, \\
& \left\langle\phi_{j i}, \varphi_{l k}\right\rangle=0 \quad j \geq l .
\end{aligned}
$$

In addition to (3).

\section{Solution of Lane-Emden Equation}

In the Wavelet- Galerkin method, the solution $u(x)$ of the equation can be approximated by the $J$ th level wavelet series on the interval $[0,1]$, by

$$
u(x)=2^{\frac{J}{2}} \sum_{k=-l+2}^{2^{J}-1} u_{J, k} \phi\left(2^{J} x-k\right)
$$

Therefore, the Galerkin discritization scheme to Eq. (1) gives a nonlinear system of equations that involves the coefficients of connection. For details about the GWM and its application for solving problem, we refer the reader to [9].

Introducing Eq. (5) into Eq. (1) we obtain

$$
\begin{aligned}
& 2^{\frac{5}{2}} \sum_{k=2-l}^{2^{J}-1} u_{k} \phi^{\prime \prime}\left(2^{J} x-k\right) \\
+ & \frac{\alpha}{x} 2^{\frac{3}{2}} \sum_{k=2-l}^{2^{J}-1} u_{k} \phi^{\prime}\left(2^{J} x-k\right) \\
+ & f\left(x, 2^{\frac{J}{2}} \sum_{k=2-l}^{2^{J}-1} u_{k} \phi\left(2^{J} x-k\right)\right) \\
= & g(x),
\end{aligned}
$$

In the other expression

$$
\sum_{k=2-l}^{2^{J}-1} u_{k} \phi_{J k}^{\prime \prime}+\frac{\alpha}{x} \sum_{k=2-l}^{2^{J}-1} u_{k} \phi_{J k}^{\prime}+f\left(x, u_{k}\right)=g(x),
$$

To determine the coefficient $u_{k}$, we take the inner product of both sides of Eq. (7) with $\phi_{J l}$, as

$$
\begin{aligned}
& \sum_{k=2-l}^{2^{J}-1} u_{k} \int_{0}^{1} \phi_{J L} \phi_{J k}^{\prime \prime} d x \\
& +\frac{\alpha}{x} \sum_{k=2-l}^{2^{J}-1} u_{k} \int_{0}^{1} \phi_{J L} \phi_{J k}^{\prime} d x+\int_{0}^{1} \phi_{J L} f\left(x, u_{k}\right) d x \\
& =\int_{0}^{1} \phi_{J L} g(x) d x, \\
& \quad L=2-l, 3-l, \ldots, 2^{J}-l .
\end{aligned}
$$

Or

$$
\begin{gathered}
\sum_{k=2-l}^{2^{J}-1} u_{k} \int_{0}^{1} \phi_{J L} \phi_{J k}^{\prime \prime} d x+\frac{\alpha}{x} \sum_{k=2-l}^{2^{J}-1} u_{k} \int_{0}^{1} \phi_{J L} \phi_{J k}^{\prime} d x \\
=\int_{0}^{1} \phi_{J L}\left(g(x)-f\left(x, u_{k}\right)\right) d x, \\
L=2-l, 3-l, \ldots, 2^{J}-1 .
\end{gathered}
$$

We assume that $\left(g(x)-f\left(x, u_{k}\right)\right)=\sum_{i=0}^{m} a_{i} x^{i}, \quad$ is a polynomial of degree $m$ in $x$.

We write the Eq. (9) as

$$
\begin{gathered}
\sum_{k=2-l}^{2^{J}-1} c_{k L}^{J} u_{k}+\frac{\alpha}{x} \sum_{k=2-l}^{2^{J}-1} b_{k L}^{J} u_{k}=d_{m L}^{J}, \\
L=2-l, 3-l, \ldots, 2^{J}-1,
\end{gathered}
$$

Where 


$$
\begin{aligned}
c_{k L}^{J} & =\int_{0}^{1} \phi_{J k}^{\prime \prime}(x) \phi_{J L}(x) d x, \\
b_{k L}^{J} & =\int_{0}^{1} \phi_{J k}^{\prime}(x) \phi_{J L}(x) d x, \\
d_{k L}^{J} & =\int_{0}^{1} \sum_{i=0}^{m} a_{i} x^{i} \phi_{J L}(x) d x .
\end{aligned}
$$
form

Equation (10) can be further put into the matrix-vector

$$
A_{1} U=D
$$

Where

$$
\begin{aligned}
& A_{1}=C+\alpha B+\beta A, B=\left[b_{k l}^{J}\right]_{2-L \leq k, l \leq 2^{J}-1}, \\
& C=\left[c_{k l}^{J}\right]_{2-L \leq k, l \leq 2^{J}-1}, D=\left[d_{k l}^{J}\right]_{2-L \leq k, l \leq 2^{J}-1}
\end{aligned}
$$

And

$$
U=\left[u_{l-2}, u_{l-3}, \ldots, u_{2} J_{-1}\right]^{t},
$$

Where $t$, denotes the matrix transpose. Now we have a linear system of $2^{J}+L-2$ equations of the $2^{J}+L-2$ unknown coefficients. We can obtain the coefficients of the approximate solution by solving this linear system. The solution $U$, gives the coefficients in the WaveletGalerkin approximation $u_{J}(x)$ of $u(x)$.

\section{Adomian Decomposition Method}

Consider the following equation

$$
y-N(y)=f,
$$

Where $N$, is a nonlinear operator from a Hilbert space $\mathrm{H}$ in to $\mathrm{H}$ and $\mathrm{f}$ is a given function in $\mathrm{H}$. Now, we are looking for $y \in H$ satisfying (14).

At the beginning of the 1980, Adomian developed a very powerful method to solve Equation (14) in which the solution $y$, was considered as the sum of decomposition series:

$$
y=\sum_{i=0}^{\infty} y_{i}
$$

And $N(y)$ as the sum of the decomposition series:

$$
N(y)=\sum_{n=0}^{\infty} A_{n} .
$$

The method consist of the following recursion scheme

$$
y_{0}=f, y_{n+1}=A_{n}\left(y_{0}, y_{1}, \ldots, y_{n}\right), n=0,1, \ldots
$$

Where the $A_{n}{ }^{\prime} \mathrm{s}$ is polynomials depending on $y_{0}, y_{1}, \ldots, y_{n}$, are called the Adomian polynomials; these are defined as

$$
n ! A_{n}=\left.\frac{d^{n}}{d \lambda^{n}}\left[N\left(\sum_{i=0}^{n} \lambda^{i} y_{i}\right)\right]\right|_{\lambda=0}, n=0,1, \ldots
$$

The Adomian technique is equivalent to determining the sequence:

$$
S_{n}=y_{1}+y_{2}+\ldots+y_{n}
$$

By using the iterative scheme

$$
S_{n+1}=N\left(y_{0}+S_{n}\right), \quad S_{0}=0
$$

Associated with the functional equation

$$
S=N\left(y_{0}+s\right)
$$

For the study of the numerical resolution (21), Cherruault used fixed point theorem [10,11,12]. In particular, we know that if $N$, is a contraction $(\|N\|<1)$ then the sequence $\left\{S_{n}\right\}$ defined by (20) converges to the only solution $S$ of (21).

Furthermore we have $y_{n}=S_{n}-S_{n-1} \rightarrow_{n \rightarrow \infty} 0$.

\section{Illustrative Examples}

To illustrate the methods two examples are provided

\begin{tabular}{|c|c|c|}
\hline$(\mathbf{X}, \alpha)$ & $\mathbf{E} \mathbf{W}$ & E decomposition \\
\hline$(0.25,0.1)$ & 4.7799E_003 & 1.6077E_009 \\
\hline$(0.5,0.1)$ & 3.8654E_003 & 2.2736E_009 \\
\hline$(0.75,0.1)$ & 2.7143E_003 & 1.6077E_009 \\
\hline$(0.25,0.5)$ & 6.5798E_002 & 5.5213E_004 \\
\hline$(0.5,0.5)$ & 7.3478E_002 & 7.8084E_004 \\
\hline$(0.75,0.5)$ & 1.8764E_002 & 5.5213E_004 \\
\hline$(0.25,0.1)$ & 3.2385E_001 & 1.2245E_001 \\
\hline$(0.5,0.1)$ & 5.7492E_001 & 1.7317E_001 \\
\hline$(0.75 .0 .1)$ & 4.2954E_001 & 1.2245E_001 \\
\hline
\end{tabular}

\subsection{Example 1}

Consider the following Lane-Emden equation

$$
\begin{aligned}
& y^{\prime \prime}(x)+\frac{\alpha}{x} y^{\prime}(x)+y(x)=6+12 x+x^{2}+x^{3}, \\
& 0<x \leq 1,0<\alpha \leq 1, \quad y(0)=0, y^{\prime}(0)=0 .
\end{aligned}
$$

The presented method is applied for $\mathrm{J}=6$.

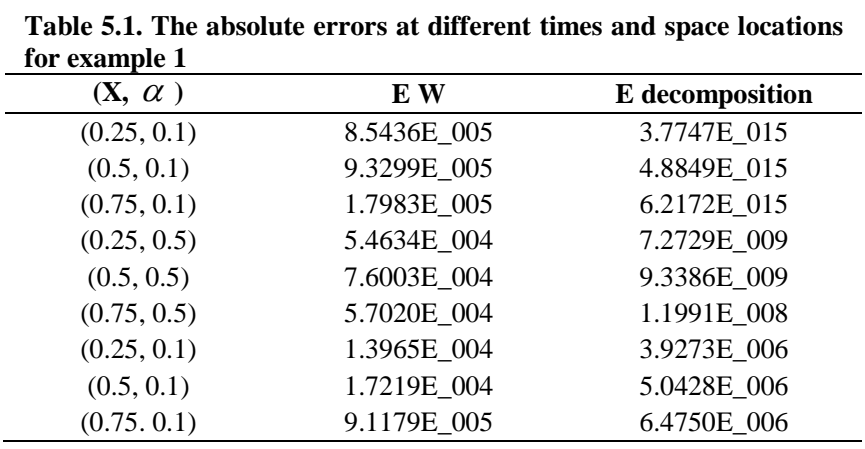

\subsection{Example 2}

Let's consider the following form of the Lane-Emden equation;

$$
\begin{aligned}
& y^{\prime \prime}(x)+\frac{\alpha}{x} y^{\prime}(x)=2(2 x+3) y(x), \\
& 0<x \leq 1, \quad 0<\alpha \leq 1 . \quad y(0)=1, \quad y^{\prime}(0)=0 .
\end{aligned}
$$

The presented method is applied for $\mathrm{J}=6$.

Table 5.2. The absolute errors at different times and space locations for example 2 


\section{Conclusion}

Many excellent properties of wavelet, such as "locality" and vanishing moments, causes the wavelet basis to be a better choice than others, in function approximation.. In other way it is seen that decomposition method can be an alternative way for the solution of partial differential equations that have no exact solutions. These two methods have been applied for Lane-Emden equation, successfully. As the results of illustrative examples show the solutions are in a good agreement with the exact ones, even up to 9 digits significant. This agreement of the solutions are so high that one can clams that these methods are a powerful numerical tools for fast and accurate solution, for such kind of differential equations.

\section{References}

[1] S. Chandrasekhar, Introduction to the study of stellar Structure, Dover, New York, 1967.
[2] H. T. Davis, Introduction to Nonlinear Differential and Integral Equations, Dover, New York, 1962.

[3] O. U. Richardson, the Emission of Electricity from Hot Bodies, Longman, Green and Co., London, New York, 1921.

[4] N. T. Shawagfeh, Nonperturbative approximate solution foe LaneEmden equation, J. Math. Phys. 34 (9) (1993) 4364.

[5] G. Adomian, Nonlinear Stochastic Operator Equations, Academic Press, San Diego, CA, 1986.

[6] A.M. Wazwaz, A new algorithm for solving differential equations of Lane-Emden type, Appl. Math. Compute. 111 (2000) 53.

[7] C. K. Chui, Wavelets: A mathematical tool for Signal Analysis, SIAM, Philadelphia, Pa, 1997.

[8] J. S. Gu, W.S. Jiang, The Haar Wavelets operational matrix of integration, Int. J. Syst. Sci. 27 (1996) 623-628.

[9] A. Avudainayagam, C. Vani, Wavelet-Galerkin method for Integro-differential equations, Appl. Math. Comput. 32 (2000) 247-254.

[10] L. Chambadal, Dictionnaire de Mathematques, Hachette, Paris, 1981.

[11] T. L. Satty, J.Bram,Nonlinear Mathematics, Mc Graw-Hill, New York, 1964.

[12] k. Abbaoui, Y. Cherruault, Convergence of Adomian's method applied to differential equations, Comput. Math. Appl. 28 (1994) 103-109. 